Ann. Génét. Sél. anim., I979, 11 (I), ro5-I10.

\title{
Modèles de répartition des populations domestiques animales après migration par vagues à partir d'un centre d'origine
}

\author{
J. J. LAUVERGNE \\ Département de Génétique animale, \\ Centre national de Recherches zootechniques, I.N.R.A., \\ 78350 Jouy-en-Josas
}

\begin{abstract}
Résumé
Pour expliquer la répartition des populations domestiques animales dans le monde, on a imaginé un modèle avec un centre d'origine d'où partent des migrations discontinues centrifuges et isotropes avec des vitesses comparables. Si 1'on admet que les races n'évoluent pas au cours de leur migration et que la dernière vague submerge génétiquement la vague précédente on aboutit à un schéma d'auréoles concentriques autour du centre d'origirie. Les auréoles portent des populations d'autant plus archaïques que l'on s'éloigne du centre. L'allure générale en auréoles emboîtées plus ou moins régulièrement est conservée lorsque les hypothèses de départ sont modifiées, au moins dans une certaine limite. Compte tenu des distorsions dues au climat et au relief cette disposition se retrouve assez bien pour les premiers Ovins de l'Ancien Monde qui auraient diffusé à partir d'un centre de domestication puis d'origine moyen-oriental.
\end{abstract}

\section{Introduction}

De nombreuses théories ont été avancées pour expliquer les débuts de la domestication des animaux (cf. IsAAC, I970, pour revue).

Certains auteurs se sont en outre attachés à expliquer la répartition des races domestiques dans le monde à l'aide des migrations (cf. par exemple Curson et 'Thornton, I936 et PAyne, I964 pour les Bovins d'Afrique).

Dans le présent article on va s'efforcer de dégager une loi de migrations susceptible d'expliquer certaines situations observées. 


\section{Genèse d'une loi de répartition simple}

Récemment nous avions été amené à définir pour les animaux domestiques la notion de "centre d'origine " (LAUVERGNE, I979). Cette notion est calquée sur les conceptions de VaviLov (I927-I928) pour les plantes cultivées.

A partir de ces centres d'origine on doit normalement envisager des migrations successives qui sont, en gros, centrifuges et vont diffuser des populations de plus en plus évoluées, tout au moins tant que le centre d'origine fonctionne.

La loi de répartition de ces races dans le plan va alors dépendre de leur comportement génétique au cours de leur migration en territoire libre et quand elles sont confrontées avec des populations déjà en place.

On peut imaginer beacoup de règles différentes auxquelles obéiraient les populations en cours de migration. Après bien des tâtonnements il nous est apparu que, pour construire un modèle le plus simple et le plus " naturel " possible, on devait en admettre trois qui sont :

- la vitesse de migration varie peu dans le temps et dans l'espace;

- les populations évoluent peu, génétiquement parlant, durant leur migration;

- la vague dernièrement arrivée remplace génétiquement la vague précédemment installée.

Pour rendre plus parlante la disposition obtenue, encore que ce ne soit pas absolument utile, on peut admettre en outre que les vagues sont discontinues.

\section{Élaboration de modèles mathématiques}

\section{I. - Modèle simplifié}

Outre les contraintes ci-dessus énumérées (vagues discontinues évoluant à des vitesses constantes et comparables, constance génétique au cours de la migration et recouvrement génétique par la dernière vague arrivante) on peut admettre que le centre d'origine a une forme circulaire et que les vagues se déplacent d'une manière strictement centrifuge et isotrope (même vitesse quelle que soit la direction dans le plan).

On peut alors adopter les notations suivantes :

Le centre d'origine est circulaire, de rayon $r$ et de centre o invariables. Sa composition génétique varie continuellement, on la note $\alpha_{0}$ à l'instant $t_{0}, \alpha_{1}$ à l'instant $t_{1} \ldots \alpha_{n}$ à l'instant $t_{n}$. Les instants $t_{0}, t_{1}, \ldots t_{n}$ sont aussi les dates de départ des vagues successives de migration qui se déplacent à des vitesses $a_{1}, a_{2}, \ldots a_{n}$.

Dans ces conditions on voit qu'à l'instant $t_{1}$ on trouve une population d'état génétique $\alpha_{0}$ dans une auréole limitée par les cercles de centre o et de rayons $r$ et $r+a_{1}\left(t_{1}-t_{0}\right)$. Cependant que, dans le centre lui-même, l'état génétique est déjà $\alpha_{1}$ (fig. $\mathrm{rB}$ ).

En $t_{2}$ la deuxième vague, de formule $\alpha_{1}$, aura recouvert une auréole limitée par le cercle de centre o et de rayon $r$ (le centre d'origine lui-même) et un cercle de rayon $r+a_{2}\left(t_{2}-t_{1}\right)$ cependant que la population de la première vague, toujours d'état $\alpha_{0}$, aura continué sa migration et se trouvera rejetée sur une auréole 
périphérique limitée à l'extérieur par le cercle de rayon $r+a_{1}\left(t_{2}-t_{0}\right)$ (fig. IC). A l'instant $t_{n}$ la répartition des populations sera constituée par une série d'auréoles emboîtées autour du centre d'origine, la plus externe étant d'état génétique le plus archaïque $\alpha_{0}$, cependant que la plus proche est d'état $\alpha_{n-1}$ (fig. I).
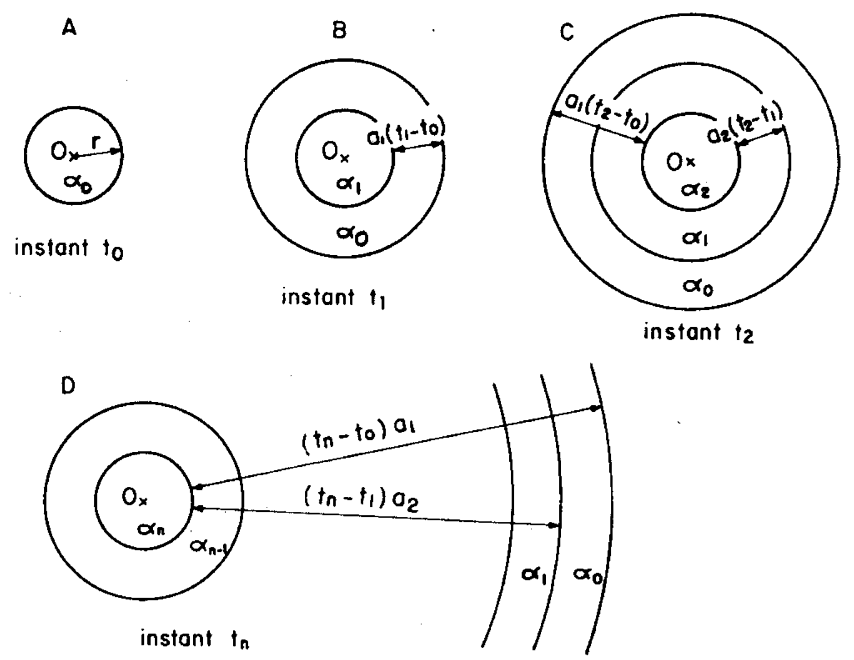

FIG. I. - Répartition des populations domestiques à divers états génétiques

dans un schéma de migrations par vagues centrifuges à partir d'un centre d'origine

A) A l'instant $t_{0}$, début de la première migration.

B) A l'instant $t_{1}$, début de la seconde migration.

C) A l'instant $t_{2}$, début de la troisième migration.

D) A l'instant $t_{n}$, début de la $n-$ I eme migration.

Distribution of domestic population with various genetic states in a diagram of migratios by centrifugal waves from a center of origin

A) At the moment $t_{0}$, start of the Irst migration.

B) At the moment, $t_{1}$, start of the 2nd migration.

C) At the moment $t_{3}$, start of the 3rd migration.

D) At the moment $t_{n}$ start of $n$ - Ith migration.

\section{2. - Modèles plus élaborés}

On imagine assez facilement - et on peut d'ailleurs le prouver mathématiquement en simulant des séries de modèles avec l'aide ordinateur — que les conditions qui nous ont permis de bâtir le modèle simplifié peuvent varier dans une certaine mesure sans que la caractéristique essentielle c'est-à-dire la disposition en auréoles emboîtées comportant dès populations de plus en plus archaïques à mesure que l'on se dirige vers la périphérie soit modifiée.

Simplement les limites des auréoles ne seront plus forcément circulaires, leur épaisseur sera variable, il pourra subsister des îlots archaïques, certaines populations présenteront des caractéristiques intermédiaires... Tout cela pour tenir compte du fait que le plan est limité par les contours des continents, que l'isotropie est 
contrariée par les variations de relief et le climat, que les populations installées peuvent se révéler mieux adaptées que les nouvelles arrivantes, que certaines initiatives de l'homme peuvent contrarier ce schéma de déplacement etc...

\section{Discussion}

La répartition des Ovins dans l'Ancien Monde, au moins avant les bouleversements qui remontent à la mérinisation, se prête assez bien à la vérification de tels modèles.

Comme nous l'avons récemment montré on dispose en effet, pour cette espèce, de marqueurs à effet visibles qui permettent de mesurer assez simplement le degré d'archaïsme d'une population ovine : composition, coloration et mue de la toison, type de cornage, conformation de la tête (chanfrein, oreilles) forme de la queue... (LAUVERGNE, I978, I979).

En outre les archéozoologues (ZEUNER, I963, CLASON, I974, DUCos, I977) aidés des caryologistes (Bunch et Foote, r977) ont localisé 1'existence d'un centre de domestication moyen-oriental qui a toutes les allures d'un centre d'origine ayant fonctionné pendant fort longtemps.

Par chance ce centre d'origine moyen-oriental a une position assez centrale dans l'Ancien Monde. Il y a donc la place pour que les auréoles se disposent avec une certaine régularité.

De fait, si 1'on compulse les catalogues des races ovines établis par Mason (1969) et TFRRILL (I979) pour le Monde entier, Mason (I967) pour le Bassin Méditerranéen, EPSTEIN et MASON (I97I) pour 1'Afrique et EPSTEIN (I969) pour la Chine on trouve bien, en gros une disposition en auréoles emboîtées.

On décèle tout d'abord à la périphérie une auréole de races archaïques, aussi bien en Scandinavie que dans les îles de l'Atlantique nord que dans 1'Afrique de 1'Ouest que dans certaines parties de la Chine : petite taille, queue courte, toison très proche du type sauvage voire entièrement poilue, à mue annuelle et grande variabilité colorée, cornage du type mouflon chez le mâle, les femelles étant sans cornes.

A l'intérieur de cette auréole se trouve une seconde auréole de races plus enlevées, souvent mottes, à chanfrein parfois bombé, à queue longue et fine, dont la toison est définitivement lainée et ne mue plus et dont la gamme des couleurs est réduite : ce sont les populations traditionnelles d'Europe Continentale (Allemagne, France, Espagne, Italie). On les retrouve en Afrique du Nord et dans le Sahel où elles sont parfois sans laine. En Asie certaines populations chinoises sont de ce type.

A l'intérieur de cette seconde auréole on rencontre ensuite les races les plus récemment produites par le centre d'origine moyen-oriental : celles qui ont la croupe ou la queue grasse. Après les enquêtes de Mason (I967) dans le Bassin Méditerranéen et de EPSTEIN et MASON (I97I) en Afrique il faudrait distinguer plusieurs zones. La diversité et la complication des types de stéatocaudie augmentent à mesure que 1'on se rapproche de la zone iranienne où SoHRABI (I937) ne distinguait pas moins de to types de queue grasse ce qui confirme le rôle ancien et encore actuel du centre d'origine moyen-oriental (Iran, Irak, Turquie).

Certaines dispositions zonées qui viennent vérifier notre modèle pour les Ovins anciens sont représentées dans la carte fournie par MAsoN (I974). L'auteur, 
toutefois utilise une classification raciale basée sur un seul critère (la queue ou la toison). Il en est de même pour TERRILL (I979) dont la très intéressante tentative de classification mondiale des races de mouton reste, pour l'essentiel monocritérielle. Pour notre propos il faudrait concevoir une sorte d'" indice d'archaîsme ".

On peut ainsi voir que l'étude de la validité du genre de modèle que nous proposons est à peine entamée. De nombreux problèmes se posent encore. Dans le cadre de cet article on doit se contenter de les énumérer. Ils concernent :

- La notion même de centre d'origine et les contraintes migratoires que nous avons admises. Il faut, semble-t-il, partir des conditions nouvelles crées par la domestication d'une espèce animale : même taux de mutation sans doute mais beaucoup plus de mutants car les effectifs sont supérieurs et leur chance d'être conservée est accrue (protection contre les prédateurs, rejet moins systématique par les congénères, coefficients de survie modifiés par la meilleure alimentation...). Le centre d'origine qui se développe, au moins dans un premier temps, en lieu et place du centre de domestication conserverait ces avantages sur les zones de colonisation récente où, les densités étant plus faibles, les contraintes bioclimatiques nouvelles moins bien maîtrisées, l'espace pas encore organisé, l'évolution se trouve en quelque sorte bloquée. Le remplacement génétique quant à lui, peut s'expliquer par le goût de la nouveauté, universellement répandu chez l'homme, mais aussi par l'avantage réel de certaines lignées nouvelles (ainsi celui du Mérinos pour produire de la laine fine).

- L'appréciation exacte des conditions qui peuvent modifier l'isotropie et empêcher le recouvrement par la population dernière arrivante. A ce propos l'étude des îlots archaïques peut être extrêmement fructueuse. Déjà les anciens auteurs avaient distingué ces populations : le mouton des Grisons (RÜTIMEYER (I86I), les races à queue courte du Nord de 1'Europe, ZEUNER (I963) et, plus récemment, la race Corse (LAUvergne et Adalsteinsson, I976). L'examen du dictionnaire de MAson (I969) montre que, dans la zone même du centre d'origine moyenoriental, il y aurait certaines zones refuge avec des populations reliques (bord Sud-Est de la Mer Noire, rivage Sud de la Caspienne).

\section{- La conception de modèles plus compliqués, voire différents :}

- lorsque des centres secondaires, en petit nombre, se mettent à fonctionner;

- lorsqu'il existe de multiples centres secondaires. Ce pourrait être le cas depuis fort longtemps pour des espèces qui ne vont pas en troupeau, comme le Chien. Plus récemment les modèles de remplacement des races envisagés par THÉRET (I975) ou DENIS (I675) semblent aussi inciter à concevoir des modèles plus diffus.

Reçu pour publication en mai 1979.

\section{Remerciements}

Les remarques de $R$. Laurans ont été des plus précieuses pour la rédaction de notre article. On n'oublie pas, non plus, les intéressantes discussions avec W. C. Foore et T. D. BuncH et leurs étudiants à l'issue d'un séminaire donnée à Logan (Utah) en mars I979. Ėnfin, on doit remercier Melinda BurRII, L, C. BRoOKE, L. OLIIVIER et N'GUYEN TAN CAQ pour leurs fructueux autant qu'amicaux commentaires. 


\section{Summary}

\section{Models of distribution for population of farm animals after migration by waves from a center of origin}

In order to explain the world partition of populations of farm animals, a simplified model has been conceived with a circular center of origin from which centrifugal isotropic migrations start wave after wave. If there are no genetic changes during the migration (genetic evolution being the privilege of the population in the center of origin), if automatically the population in the field is genetically overwhelmed by the latter arriving wave and if the speeds of migration are comparable from wave to wave one obtains a design with circular concentric halos. The halos carry populations which are more and more archaic as one goes away from the center of origin. This general feature of encased halos is kept even when the basic conditions are not all exactly fullfilled. The previous distributions of sheep in the Old World are explained in a reasonably way with such models when one assumes centers of origin in the Middle East.

\section{Références bibliographiques}

Bunch T. D., Foote W. C., I978. Cytogenetic evidence on the ancestral stock of domestic sheep (Ovis aries). Ethnozootechnie, 21, II-I5.

Cr,ason A. J., r974. Archaeozoological research and the earliest stock-breeding in the Near East. Eastern Anthropol., 27, 5-21.

Curson H. H., Thornton R. W., r936. A contribution to the study of African Native cattle. Onderstepoort J. Vet. Sci. anim. Indust., 7, 6r3-739.

Denss B., I975. Conséquences génétiques de l'évolution des races. Bull. tech. Dép. Génét. anim. (I.N.R.A.), 20, $12-18$.

Ducos P., 1978. Les débuts de l'élevage du mouton au Proche-Orient. Ethnozootechnie, 21, 33-36.

EPSTEIN H., 1969. Domestic animals of China. Commonwealth Agricultural Bureaux, Farnham Royal Bucks. England 33-57, 69-33.

EPSTEN H., Mason I. L., I97I. The origin of the domestic animals of Africa. Africana Publishing corporation, New-York, London, Munich, 2 vol. 573 and 719 p.

ISAAC E., I970. Geography of domestication. Prentice-Hall, Englewood Cliffs, 3-i6.

LAUVERGNE J. J., I979. Visible genetic markers in domestic sheep and their ancestors, in Foore W. C., Bunch T. D. The domestication of Sheep, International Sheep and Goat Institute, Logan, Utah, 2 I-23.

LAUVERGNE J. J., I978. Utilisation des marqueurs génétiques pour l'étude de l'origine et de l'évolution du mouton domestique. Ethnozootechnie, 21, 1 7-23.

r.AUVERGNe, J. J., Adarsteinsson, 1976. Gènes pour la couleur de la toison de la brebis Corse. Ann. Génét. Sél. anim., 8, I53-I 72.

MASON I. L., r967. Sheep breeds of the Meditervanean. F.A.O. and C.A.B., Rome and Farnham Royal, Bucks. England.

Mason I. L., I969. A Dictionary of Livestock Breeds. Commonwealth Agricultural Bureaux, Farnham Royal, Bucks, England, 268 p.

Mason I. L., I974. The role of natural and artificial selection in the origin of breeds of farm animals $Z$. Tievzücht. Zucht Biol., 90, 229-244.

Payne W. J. A., I964. The origin of domestic cattle in Africa. Emp. J. Exper. Agric. 32, 97-I 3. RÜTIMEYER, I86r. Cité par ZEUNER (I963).

Sohrabi S., I937. Les races de mouton et la laine en Iran. Thèse Fac. Sci. Univ. Paris, I 86 p.

TERRILI C. E., I979. The distribution of breeds of sheep as related to domestication and development of modern genotypes, in Foote W. C., BUNCH T. D. The domestication of Sheep, International Sheep and Goat Institute, Logan, Utah, 4I-II5.

Thúret M., r975. Essai d'une dynamique des races. Bull. tech. Dép. Génét. anim., I.N.R.A., 20, 4-I I.

VAVILOV N. I., I927-1928. Geographische Cenzentren unserer Kulturpflanzen Leipzig.

ZEUNER F. E., 1963. A History of domesticated Animals. Hutchinson, London, I 53-244. 